\title{
The Effects of Temporary Inactivation of the Core and the Shell Subregions of the Nucleus Accumbens on Prepulse Inhibition of the Acoustic Startle Reflex and Activity in Rats
}

\author{
Helen HJ Pothuizen', Ana L Jongen-Rêlo ${ }^{1,2}$ and Joram Feldon*,1 \\ 'Laboratory of Behavioural Neurobiology, Swiss Federal Institute of Technology Zurich, Schwerzenbach, Switzerland
}

\begin{abstract}
The nucleus accumbens can be dissociated into at least two subregions: a 'core' and a 'shell'. Using temporary chemical inactivation of these subregions, we investigated whether they are differentially involved in the regulation of prepulse inhibition (PPI) of the acoustic startle reflex and activity. For this purpose, rats were bilaterally implanted with guide cannulae aimed at either the core or the shell and infused with the GABA $\mathrm{G}_{\mathrm{A}}$ receptor agonist muscimol $(0.5 \mu \mathrm{g} / 0.2 \mu \mathrm{l}$ per side). The control group consisted of vehicle infused and unoperated rats. To ascertain the region selectivity of the infusions, $0.2 \mu \mathrm{l}$ of $\left[{ }^{3} \mathrm{H}\right]$ muscimol was infused into either the core or the shell of an additional group of rats. The behavioral results demonstrated that in comparison to the control group, inactivation of the core led to a loss of the prepulse intensity dependency of PPI. Moreover, core inactivation resulted in akinesia directly after infusion, but in hyperactivity 24 and $72 \mathrm{~h}$ thereafter in contrast to the control group. In both experiments, inactivation of the shell was ineffective compared to controls. Analysis of the autoradiograms revealed that the spread of drug into the other subregion was minimal, supporting the region selectivity of the inactivation. These results lend further support to the existence of a functional dissociation between the core and the shell, with the former being preferentially involved in PPI and locomotion. The persistent hyperactivity after the muscimol infusion into the core could be explained by compensatory mechanisms taking place in the nucleus accumbens.

Neuropsychopharmacology (2005) 30, 683-696, advance online publication, 19 January 2005; doi: I 0.1038/sj.npp. 1300643
\end{abstract}

Keywords: ventral striatum; accumbens; $G_{A B A}$ receptor; microinfusion; locomotor activity; sensorimotor gating

\section{INTRODUCTION}

It has become evident that the nucleus accumbens does not constitute one functionally uniform structure. Several studies in the rodent brain employing permanent lesion techniques as well as intracerebral pharmacological manipulations have revealed a functional dissociation between the subregions of the nucleus accumbens: the 'core' and the 'shell' (as originally defined by Záborszky et al, 1985). Whereas the core subregion has been demonstrated to be preferentially involved in the mediation of motor behavior (Johnson et al, 1996; Maldonado-Irizarry and Kelley, 1994; Parkinson et al, 1999), the shell subregion tends to be more

*Correspondence: Dr J Feldon, Behavioral Neurobiology Laboratory, Swiss Federal Institute of Technology Zürich, Schorenstrasse 16, CH-8603 Schwerzenbach, Switzerland, Tel: +4l | 65574 48, Fax: +4I I 65572 03, E-mail: feldon@behav.biol.ethz.ch

${ }^{2}$ Current address: Neuroscience Research, Department of Pharmacology, Abbott GmbH \& Co. KG, Knollstrasse, 6706I Ludwigshafen, Germany.

Received 21 July 2004; revised 10 November 2004; accepted II November 2004

Online publication: 12 November 2004 at http://wmw.acnp.org/citations/ NPPI | | 204040330/default.pdf closely associated with motivational and attentional behavior (Jeanblanc et al, 2002; Weiner et al, 1996). We have recently confirmed and extended these findings by showing that selective $\mathrm{N}$-methyl-D-aspartic acid (NMDA)-induced excitotoxic lesions of either the core or the shell result in differential effects on locomotor activity, latent inhibition assessed with two-way active avoidance, spatial working memory, and prepulse inhibition (PPI) of the acoustic startle reflex (Jongen-Rêlo et al, 2002, 2003).

Supporting evidence for the notion that the core and the shell might constitute two separate functional domains comes from anatomical findings, which demonstrate clear differences in the afferent and efferent projections of the two subregions in the rodent brain (Brog et al, 1993; Groenewegen et al, 1999; Heimer et al, 1991; Zahm and Brog, 1992; Zahm and Heimer, 1990). Furthermore, the two subregions are distinct in their neurochemical content, in the distribution of various receptor types and in their neuronal substrates (eg Deutch and Cameron, 1992; Jones et al, 1996; Jongen-Rêlo et al, 1993, 1994; O'Donnell and Grace, 1993; Záborszky et al, 1985). Due to its predominant innervation by limbic structures and its preferential involvement in 'limbic'-associated behavioral functions, the shell region is generally referred to as the 'limbic part' 
of the accumbens, whereas the core is regarded as the ventral extension of the dorsal striatum or the 'motor sector' of the nucleus accumbens.

PPI refers to the reduction of startle reaction to a startleeliciting stimulus when it is shortly preceded by a weak stimulus (Hoffman and Searle, 1965). It is considered to be an operational measure of sensorimotor gating since the preceding weak stimulus triggers a protective or 'gating' mechanism that limits the processing of, and therefore the motor response to, the succeeding intense stimulus. Studies employing lesions or pharmacological manipulations of the nucleus accumbens in rats have shown that this structure seems to have a crucial role in the regulation of PPI (eg Jongen-Rêlo et al, 2002; Kodsi and Swerdlow, 1994, 1997; Wan et al, 1995; Wan and Swerdlow, 1996). The nucleus accumbens appears to exert this regulatory role by virtue of its GABAergic output to the ventral pallidum and its projection to the pedunculopontine nucleus, providing a direct link to the primary startle circuit (Kretschmer and Koch, 1998). At the same time, it receives modulatory input via glutamatergic projections from 'limbic' structures (such as the hippocampus, medial prefrontal cortex, amygdala, and cingulate gyrus) and via dopaminergic projections from the ventral tegmental area (reviewed by Swerdlow et al, 2001).

Several studies have addressed the question whether the core or the shell is preferentially involved in the regulation of PPI or if both subregions contribute to this equally, but possibly in a different manner (Jongen-Rêlo et al, 2002; Kodsi and Swerdlow, 1997; Wan et al, 1994; Wan and Swerdlow, 1996). The general picture emerging from these studies suggests that the regulatory effects of the two subregions on PPI are not as clearly demarcated as are, for example, their distinct anatomical projection patterns. Infusion of the dopamine $\mathrm{D}_{2} / \mathrm{D}_{3}$ receptor agonist quinpirole into either the core or the shell resulted in a dose-dependent reduction of PPI (Wan et al, 1994). In addition, electrolytic lesions of the core as well as the shell are able to significantly reduce PPI in rats (Kodsi and Swerdlow, 1997; but see also Jongen-Rêlo et al, 2002). Although both subregions seem to be involved in the regulation of PPI, they do show a prominent diversity in their neurochemical regulation of sensory motor gating (Wan and Swerdlow, 1996).

Up to now, the relative contribution of the two subregions to the modulation of locomotor activity is still unclear. Kelley and co-workers have shown that lesions as well as pharmacological interventions of the core result in changes of spontaneous locomotor activity, whereas these manipulations were ineffective when applied to the shell (Maldonado-Irizarry and Kelley, 1994, 1995). Similar findings were reported by Johnson et al (1996) and Parkinson et al (1999). Furthermore, the core subregion appears to be the site of action for psychostimulant (such as amphetamine or nicotine)-induced locomotor activity (Boye et al, 2001; Parkinson et al, 1999; Sellings and Clarke, 2003; but see Weiner et al, 1996).

Yet, our own recent findings as well as data of others indicate that the apparently less significant role of the shell subregion in locomotor activity remains ambiguous. Pharmacological blockade of NMDA neurotransmission in the shell using the NMDA receptor antagonist DL-2-amino- 5-phosphonopentanoic acid (AP5, Pulvirenti et al, 1994), electrolytic lesions of the shell in combination with systemic saline injections (Weiner et al, 1996), as well as excitotoxic lesions restricted to the shell (Jongen-Rêlo et al, 2002), all resulted in changes in locomotor activity. In contrast, these manipulations were ineffective when applied to the core.

The controversies described in the published data listed above could be explained by methodological differences, which are inherent to the use of either permanent lesions or pharmacological manipulations. In contrast to more commonly used permanent lesion techniques, the present study employed a carefully monitored temporary inactivation technique using microinfusions of the $\gamma$-aminobutyric acid $(\mathrm{GABA})_{\mathrm{A}}$ receptor agonist muscimol to investigate the involvement of the core and the shell in PPI and locomotor activity. In contrast to most of the published studies, we have determined the extent of diffusion of a small volume of muscimol into either the core or the shell prior to the behavioral experiments in order to assure the regional selectivity of the microinfusion technique. Muscimol microinfusion has demonstrated to be an efficient and potent technique to transiently inactivate discrete brain structures (Bast et al, 2001; Martin and Ghez, 1999), and its use exhibits several advantages above permanent lesion techniques. First, although the overall action of muscimol is brief, it has a rapid onset (Martin and Ghez, 1999). Second, it offers fewer opportunities for compensatory changes to occur in the brain than permanent lesions (see also Bast and Feldon, 2003). Moreover, as a result of the short-lasting character of the manipulation, it facilitates within-subject comparisons. Given this latter feature, we were able to evaluate the relative contribution of the core or the shell in specific aspects of the behavioral processes studied.

\section{MATERIAL AND METHODS}

\section{Subjects}

Male Wistar rats $(n=38$, bred at the animal facilities of the Behavioural Neurobiology Laboratory, Schwerzenbach, Switzerland), weighing $280-300 \mathrm{~g}$ at the time of surgery, were housed under temperature $\left(21 \pm 1^{\circ} \mathrm{C}\right)$ and humidity $(55 \pm 5 \%)$ controlled conditions. The animals had free access to food chow (Kliba 3430, Klibamühlen, Kaiseraugst, Switzerland) and water throughout the experimental period. The rats were housed in groups of four (Makrolon IV) and kept under a reversed $12 \mathrm{~h}$ light/dark cycle (lights on 1900700). After surgery the rats were individually caged. All behavioral tests were conducted during the dark phase. The animals were handled daily for at least 1 week before surgery.

The Swiss Federal Veterinary Office approved all procedures used in this study, and any effort has been made to minimize the number of animals used.

\section{Guide Cannulae Implantation}

The rats were randomly assigned to one of the three groups; core-implanted rats $(n=16)$, shell-implanted rats $(n=16)$ or unoperated controls $(n=6)$. Prior to surgery, the rats were anesthetized with sodium pentobarbital $(50 \mathrm{mg} / \mathrm{kg}$, i.p., Nembutal, Abbott Laboratories, IL) and placed in the 
stereotaxic frame (David Kopf Instruments, Tujunga, CA) with bregma and lambda positioned in the same horizontal plane. An incision was made along the midline of the scalp and the skin was deflected. A pair of guide cannulae ( $9.0 \mathrm{~mm}$ long, 26 gauge, stainless steel) held by a Perspex holder was implanted through small holes $(1.5 \mathrm{~mm}$ diameter) drilled on each side of the skull. The tips of the guide cannulae were aimed at the following coordinates: for the core region $1.2 \mathrm{~mm}$ anterior from Bregma, $\pm 2.0 \mathrm{~mm}$ from the midline, and $6.5 \mathrm{~mm}$ below the dura; and for the shell region $1.2 \mathrm{~mm}$ anterior from Bregma, $\pm 0.9 \mathrm{~mm}$ from the midline, and $6.5 \mathrm{~mm}$ below dura.

The guide cannulae were anchored to the skull with three small stainless screws and secured with dental cement (Paladur, Heraeus Kulzer Gmbh \& Co., Wehrheim, Germany). Stainless steel stylets (34 gauge), which extended $0.5 \mathrm{~mm}$ beyond the tips of the guide cannulae, were placed inside it to prevent occlusion. After completion of the surgery, the animals' postsurgery behavior was monitored for at least $3 \mathrm{~h}$. The rats were allowed to recover for 7 days prior to behavioral testing. During this time, the animals were handled daily and any lost stylets were replaced.

\section{Intra Accumbens Microinfusion}

On the day of infusion, muscimol $\left(\mathrm{C}_{4} \mathrm{H}_{6} \mathrm{~N}_{2} \mathrm{O}_{2}\left[1 / 2 \mathrm{H}_{2} \mathrm{O}\right]\right.$, Tocris, Bristol, UK) was dissolved into $0.9 \%$ saline solution (Braun Medical AG, Emmenbrücke, Switzerland) at a concentration of $2.5 \mu \mathrm{g} / \mu \mathrm{l}$.

The rats were manually restrained and the stylets were removed and replaced with 34 gauge stainless steel infusion cannulae, which extended $0.8 \mathrm{~mm}$ below the tip of the guide cannulae. The infusion cannulae were connected via flexible polyetheretherketone (PEEK) tubing to $10 \mu \mathrm{l}$ Hamilton microsyringes. The microsyringes were mounted on a microinfusion pump (World Precision Instruments, Sarasota, FL).

Muscimol or $0.9 \%$ saline vehicle solution was infused bilaterally at a rate of $0.5 \mu \mathrm{l}$ per min. The injected volume was $0.2 \mu \mathrm{l}$ per side. At the end of infusion, the infusion cannulae were left in place for an additional minute to allow for diffusion before being retracted. Subsequently, the stylets were replaced and immediately after this behavioral testing commenced.

\section{Behavioral Testing}

\section{Prepulse inhibition.}

Apparatus: PPI was assessed in four sound attenuated startle chambers (SR-LAB, San Diego Instruments, San Diego, CA), which were illuminated and ventilated. Each chamber contained a transparent Plexiglas tube (diameter $8.2 \mathrm{~cm}$, length $20 \mathrm{~cm}$ ) mounted on a Plexiglas frame. Acoustic noise bursts were presented via a speaker mounted $24 \mathrm{~cm}$ above the tube. A piezoelectric accelerometer mounted below the frame detected and transduced motion within the tube. Throughout the session a background noise level of $68 \mathrm{~dB}$ [A] was maintained. Startle amplitudes were defined as the average of $1001-\mathrm{ms}$ stabilimeter readings collected from stimulus onset. The sensitivity of the piezoelectric accelerometer was routinely calibrated to ensure consistency between chambers and across sessions.
Experimental procedure: All rats were tested in the PPI experiment and were allocated to the following treatment groups: 'Core-MUS' (core-implanted rats infused with muscimol; $n=11$ ), 'Shell-MUS' (shell-implanted rats infused with muscimol; $n=11$ ), vehicle infused ( 5 core- and 5 shell-implanted rats) and unoperated rats $(n=6)$. Rats were run in squads of four with chamber positions counterbalanced across the different treatment groups.

On the first day ('baseline'), the rats were tested for startle and PPI. After matching of their basal startle and PPI values, the rats were allocated to either the drug or the vehicle infused groups. On the second day ('infusion'), the rats were infused with muscimol $(0.5 \mu \mathrm{g} / 0.2 \mu \mathrm{l}$ per side) or vehicle $(0.2 \mu$ l per side) immediately before the PPI test. On the third day ('re-baseline'), the rats were tested again without infusion to check for possible long-term effects of the infusion. The procedure of PPI testing was identical across all 3 days. On each day, the rats were placed into the PPI chamber for a 5-min acclimatization period with a 68$\mathrm{dB}[\mathrm{A}]$ background noise level that continued throughout the session. After the acclimatization period, four startle pulses of $120-\mathrm{dB}$ [A] broad band bursts of $30 \mathrm{~ms}$ were presented to test for startle responsiveness (habituation trials). Subsequently, six blocks of 11 trials were presented to assess PPI. Each block included four different trial-types presented pseudo-randomly (with an intertrial interval of 10-20s), consisting of pulse alone (two trials), prepulse alone (one trial for each prepulse intensity), prepulse followed by a pulse $100 \mathrm{~ms}$ after prepulse onset (one trial for each prepulse intensity), and no stimulus (one trial). The prepulse ( $20 \mathrm{~ms}$ of broad band burst) had an intensity of either $72,76,80$, or $84 \mathrm{~dB}$ [A] (ie $4,8,12$, and $16 \mathrm{~dB}$ above the background).

\section{Activity.}

Apparatus: The apparatus consisted of 16 activity chambers $(25 \mathrm{~cm}$ wide $\times 40 \mathrm{~cm}$ long $\times 40 \mathrm{~cm}$ high $)$. Each chamber was composed of three Plexiglas walls and one wooden side wall and was placed in a sound attenuated wooden cubicle. The floor of each chamber consisted of a black removable tray in which a thin layer of dark, absorbent, autoclaved soil was placed. The ceiling of each chamber was open. A fan mounted on the back wall of each cubicle provided ventilation and background noise. A monochrome video camera with a wide angle $\left(100^{\circ}\right)$ 2.5mm lens (VPC-465B; CES AG, Zurich, Switzerland) centered approximately $49 \mathrm{~cm}$ above the floor of the chamber was mounted in the ceiling of each cubicle. Six infrared $(875 \mathrm{~nm})$ light-emitting diodes (HSDL-4220; Hewlett Packard) positioned in the ceiling of each cubicle provided sufficient light for the camera to detect the rat. Images provided by each of the 16 cameras were integrated into a 16-part single image (400000 pixels) by a 16-channel multiplexer (Sony model YS-DX216CE), and the image was recorded by a video-recorder (SVT1000, Sony). The multiplexer was connected to a Dell computer (OptiPlex GXpro with a Pentium Pro Processor) running image analysis software. The software was a custom-written Visual Basic Program (Peter Schmid) that was based on an NIH Image Analysis script. Every second an image was 'taken' from each chamber and then compared pixel by pixel with 
the image obtained in the preceding second. Since each rat was monitored against a dark background, the percentage of pixels changed between successive seconds could be calculated. This percentage provided a measurement of the animal's movement or 'activity'. One-second activity values ranged from $0 \%$ (no movement) to approximately $7.5 \%$.

Experimental procedure: The activity experiment was conducted 1 week after the PPI experiment. All rats subjected to the PPI assessment were tested again here. Hence, the cannulae implanted rats had received one intracerebral infusion previously. These rats were counterbalanced across the previous treatment and reassigned to either the muscimol or the vehicle group prior to the activity experiment. The following treatment groups were used: 'Core-MUS' (core-implanted rats infused with muscimol, $n=11$ ), 'Shell-MUS' (shell-implanted rats infused with muscimol, $n=11$ ), vehicle infused (5 core- and 5 shellimplanted rats) and unoperated rats $(n=6)$.

The experiment lasted 4 days. On the first day ('baseline'), the basal activity was measured over $90 \mathrm{~min}$ without infusion of the drug. After matching of their basal activity values, the rats were allocated to either the muscimol or the vehicle infused groups. On the following day ('infusion'), the activity was measured immediately after the drug infusion to compare the acute effects of muscimol infusion $(0.5 \mu \mathrm{g} / 0.2 \mu \mathrm{l}$ per side) with saline infusion $(0.2 \mu \mathrm{l}$ per side $)$ in a between-subject design. At $24 \mathrm{~h}$ ('re-baseline 1') and $72 \mathrm{~h}$ after the infusion ('re-baseline day'), the basal activity was measured again.

\section{Data Analysis}

All statistical analyses were performed using the statistical software StatView 5.01 (SAS Institute Inc., Cary, NC) implemented on a PC running the Microsoft Windows XP operating system. As preliminary analyses showed that in both the PPI and the activity experiments, the vehicle infused and the unoperated rats did not differ statistically from each other, they were combined into one control group ('control') for the final statistical analyses reported here. Significant main effects or interaction terms were further investigated by post hoc comparisons using Fisher's protected least significance test. Statistical significance was set at a probability level of $p<0.05$ for all tests.

PPI experiment. The startle reactivity to the four pulsealone trials at the beginning of the session ('startle habituation') was submitted to a $3 \times 3 \times 4$ analysis of variance (ANOVA) consisting of a between-subjects factor of treatment (Core-MUS, Shell-MUS and control) and a repeated measurements factor of days (3) and pulse-alone presentation (4). A $3 \times 3 \times 12$ ANOVA consisting of a between-subjects factor of treatment and a repeated measurements factor of days and pulse-alone presentation (12) was conducted on the startle reactivity during the following 12 pulse-alone trials.

The reactivity on the no-stimulus and prepulse-alone trials were submitted to a $3 \times 3 \times 5$ ANOVA with a betweensubject factor of treatment ( 3 ) and a repeated measurements factor of days (3) and prepulse intensity (5). A natural logarithmic transformation was carried out before the data were submitted to the analysis, in order to better conform to the normality and variance homogeneity assumption of parametric ANOVA.

The percentage PPI (\%PPI) induced by each of the prepulse intensities was calculated using the following formula: $100-(100 \times($ startle reactivity on prepulse plus pulse trials/startle reactivity on pulse-alone trials)). The $\%$ PPI was submitted to a $3 \times 3 \times 4$ ANOVA consisting of a between-subjects factor of treatment and repeated measurements factors of days (3), and of prepulse intensity (4).

In case the aforementioned ANOVAs yielded a significant high-order interaction with treatment and days, the dependent variables were submitted to restricted ANOVAs separately for each experimental day.

Activity experiment. The 1-s activity values obtained from the computer were averaged into 10 -min values, and this measure was subjected to a $3 \times 3 \times 9$ ANOVA with a between-subjects factor of treatment condition (Core-MUS, Shell-MUS, and control) and a repeated measurements factor of days (3) and 10-min blocks (9). In case this analysis revealed a significant high-order interaction between treatment and days, the activity on each of the experimental days was analyzed separately using a $3 \times 9$ ANOVA with a between-subjects factor of treatment and a repeated measure of block.

\section{Histology}

At the end of behavioral testing, the rats were anesthetized with sodium pentobarbital $(60 \mathrm{mg} / \mathrm{kg}$, i.p.). Subsequently, they were perfused with $0.9 \%$ natrium chloride solution (Fluka, Buchs, Switzerland) at room temperature for $2 \mathrm{~min}$ (flow rate $35 \mathrm{ml} / \mathrm{min}$ ), followed by a $4 \%$ solution of freshly depolymerized paraformaldehyde in $0.1 \mathrm{M}$ phosphate-buffered saline (PBS) at $4{ }^{\circ} \mathrm{C}(\mathrm{pH} \mathrm{7.2)}$ for $15 \mathrm{~min}$ at a flow rate of $35 \mathrm{ml} / \mathrm{min}$. The brains were removed from the skull and postfixed for $48 \mathrm{~h}$ in the fixative. The brains were cryoprotected in a $30 \%$ sucrose solution (in $0.1 \mathrm{M}$ PBS, $\mathrm{pH} 7.4$, Fluka) for 2 days at $4{ }^{\circ} \mathrm{C}$ under gentle agitation.

Five adjacent series of $40 \mu \mathrm{m}$ coronal sections were cut frozen on a sliding microtome. Four series were collected into a cryoprotectant solution (30\% ethylene glycol, $25 \%$ glycerol in $50 \mathrm{mM} \mathrm{PB}$, Fluka, $\mathrm{pH} 7.4$ ), and stored at $-20^{\circ} \mathrm{C}$ until used. The remaining series were directly collected onto gelatin-coated slides and stained with cresyl violet. After staining, the sections were dehydrated through an alcohol series, cleared with xylene, and cover slipped with Eukitt (Kindler, Freiburg, Germany).

The sections were examined with the aid of a Zeiss Axiophot microscope (magnification $\times 10$ ) and the location of the guide cannulae was verified using the atlas of Paxinos and Watson (1997) as reference.

\section{$\left[{ }^{3} \mathrm{H}\right]$ muscimol Infusion}

The major aim of the present study was to functionally dissociate the core and the shell subregions of the nucleus accumbens. Hence, it was crucial for the pharmacological manipulation to be selective for either subregion. Therefore, in order to determine the extent to which the diffusion of 
muscimol is restricted to the core or the shell, the actual spread of the infused muscimol into the subregions was examined by infusing radioactively labeled muscimol using the same protocol as to be used for the activity- and PPI measurements, prior to the behavioral experiments. For this purpose, an additional four core- and three shell-implanted rats were used. The $\left[{ }^{3} \mathrm{H}\right]$ muscimol (tritium labeled muscimol) $(1 \mu \mathrm{Ci} / \mu \mathrm{l}, 517 \mathrm{Ci} / \mathrm{mmol}$, Amersham Life Sciences, Dübendorf, Switzerland) was mixed with unlabeled muscimol (Tocris) to give a final concentration of $2.5 \mu \mathrm{g} / \mu \mathrm{l}$. The animals were infused with $0.2 \mu \mathrm{l}$ of $\left[{ }^{3} \mathrm{H}\right]$ muscimol and decapitated after $15 \mathrm{~min}$. The brains were quickly removed from the skulls and immediately frozen in isopentane $\left(-60^{\circ} \mathrm{C}\right)$, then cut on a cryostat into five adjacent series of $20 \mu \mathrm{m}$-coronal sections. The sections were thaw mounted onto gelatin coated slides. The slides were exposed to BioMax film (Kodak) for 10.5 weeks. Calibrated $\left[{ }^{3} \mathrm{H}\right]$ standards were incorporated in all cassettes to allow quantification of the signal. Thereafter, the slides were counterstained with cresyl violet to ascertain the cannulae location.

\section{Quantification of Autoradiograms}

Digital images of the autoradiograms were obtained using a video camera (Megaplus Model 1.4I, Kodak, San Diego, CA) and the software package Image-pro Plus (version 4.0, Media Cybernetics, LP, Silver Spring, MD). Using the software, the area of labeled signal was delineated on the digitally acquired images, and the following parameters from each labeled area were measured: (a) optical density, (b) width (medial-lateral extent), (c) height (dorsal-ventral extent) and (d) surface area (cf Edeline et al, 2002; Martin, 1991). All sections (on average 23 per brain) covering the total injection site along its rostral-caudal axis were measured, yielding a final section interval of $100 \mu \mathrm{m}$. The rostrocaudal extent of diffusion was estimated by measuring the distance between the injection track (as determined by the Nissl stained sections) and the last section on which the image analysis system detected labeling above background level. The optical densities were converted into $\mathrm{kBq} / \mathrm{g}$ polymer based on the standard curve calculated from the $\left[{ }^{3} \mathrm{H}\right]$ standards and subtracted from the optical density measured from the film background to standardize the values.

\section{RESULTS}

\section{Histological Evaluation of the Cannulae Placements}

Two shell-implanted rats acquired serious infections and died shortly after the first re-baseline test of the activity experiment; they were therefore discarded from the behavioral analysis. Evaluation of the location of the injection site after histology revealed that in two coreimplanted and two shell-implanted rats, the infusion site was not in the target area. The data of these rats were also excluded from the analysis. The final numbers of subjects in the Core-MUS, Shell-MUS, vehicle infused, and unoperated groups were 9, 7, 9, and 6 in the PPI experiment, and $11,7,8$, and 6 in the activity experiment, respectively (out of a total of 14 core- and 12 shellimplanted rats). A summary of the location of the cannulae tips in the core- and the shell-implanted brains is depicted in Figure $1 \mathrm{a}$ and $\mathrm{b}$, respectively.

A coronal cresyl violet stained section of a representative core-implanted brain is depicted in Figure 2a. The coreimplanted brains were characterized by extensive gliosis around the cannula tract in the neocortex and caudate putamen and some minor gliosis in the most dorsal parts of the core subregion. No damage was apparent in the shell subregion of the nucleus accumbens. As a general feature of the core-implanted brains, the lateral ventricles were slightly enlarged in comparison to the unoperated brains.

A coronal stained section of a representative shellimplanted brain is depicted in Figure 2b. The shellimplanted brains were characterized by substantial gliosis around the cannulae tract in the neocortex, the lateral septum and some minor gliosis in the most dorsal part of the shell subregion. In all cases, no damage was evident in the core subregion.

\section{General Behavioral Effects of Muscimol Infusion into the Nucleus Accumbens}

Close observation of the rats directly after the drug infusion revealed that muscimol infusion into the shell subregion resulted in enhanced jaw movements and marked stimulation of feeding behavior. These effects did not last for more than a few hours and were not observed after muscimol infusion into the core subregion. Similar observations in feeding behavior after infusion of muscimol into the shell subregion have been reported previously (eg Stratford and Kelley, 1997). In contrast, rats infused with muscimol into the core were akinetic directly after the infusion. This massive and immediate reduction in motility (see also the results of the activity experiment) disappeared $2-3 \mathrm{~h}$ after infusion and was not seen after muscimol infusion into the shell.

\section{Effects of Muscimol Infusion on PPI}

Startle habituation. Analysis of the average startle during the first four pulse alone presentations ('habituation trials') across the three experimental days did not reveal any significant treatment $(\mathrm{F}(2,28)=0.77, p>0.47)$, or day effect $(\mathrm{F}(2,56)=1.07, p>0.34)$, nor a treatment $\times$ day interaction $(\mathrm{F}(4,56)=0.64, p>0.63)$. The analysis did reveal a significant main effect of 'four pulses' $(\mathrm{F}(3,84)=5.78$, $p<0.0013)$, reflecting a short-term habituation of the startle reactivity to the four pulses (see Figure $3 \mathrm{a}$ for data on the infusion day). Its interaction with treatment did not attain significance $(\mathrm{F}(6,84)=0.51, p>0.79)$.

Startle reactivity. A $3 \times 3 \times 12$ ANOVA carried out on the startle reactivity on the 12 pulse-alone trials (following habituation) across the days yielded no significant treatment $(\mathrm{F}(2,28)=0.73, p>0.49)$, or day effect $(\mathrm{F}(2,56)=1.50$, $p>0.23)$, nor an interaction between the two factors $(\mathrm{F}(4,56)=0.51, p>0.73)$. The effect of the 12 pulse-alone trials did attain significance $(\mathrm{F}(11,308)=12.34, p<0.0001)$, which again reflects short-term habituation of the startle reaction to the pulse stimulus (see Figure $3 \mathrm{~b}$ for data on the infusion day). Its interaction with treatment was not significant. 

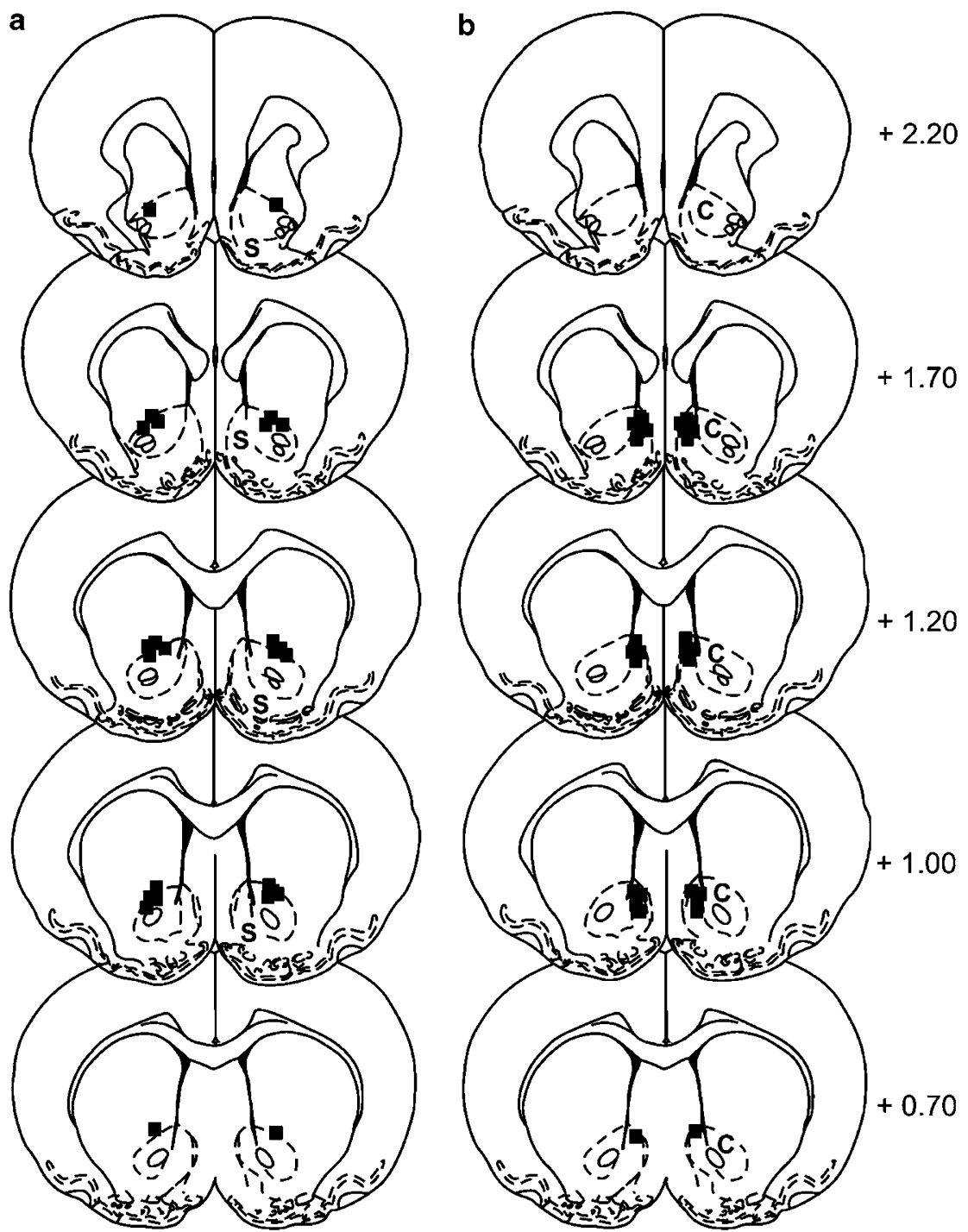

Figure I Schematic representations of the location (indicated with black squares) of the guide cannulae tips in the core ' $\mathrm{C}$ ' (a) and the shell ' $\mathrm{S}$ ' (b) of the nucleus accumbens, respectively. The numbers refer to millimeters anterior to Bregma according to the rat brain stereotaxic atlas (Paxinos and Watson, 1997).
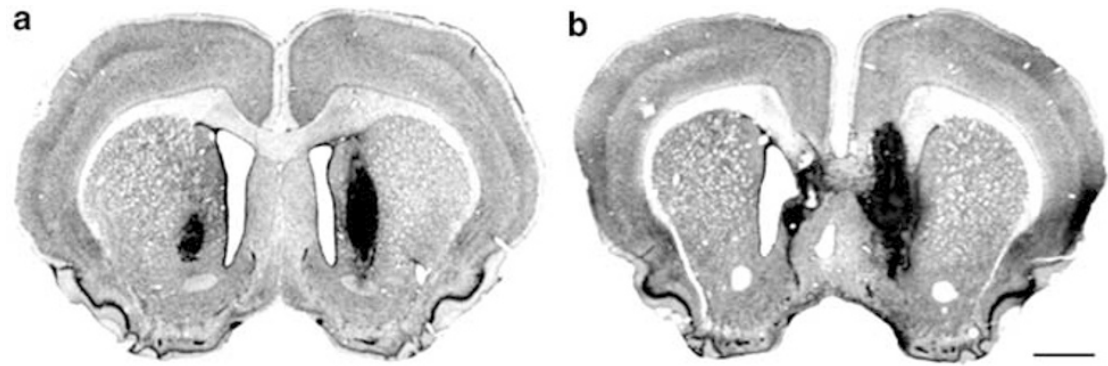

Figure 2 Photomicrographs of cresyl violet stained coronal sections through the nucleus accumbens illustrating the localization of the guide cannulae in the core (a) and the shell (b) subregion. Scale bar applies to both pictures and represents $1.5 \mathrm{~mm}$.

Prepulse alone. The $3 \times 3 \times 5$ ANOVA of the reactivity on the no stimulus and prepulse-alone trials across the 3 days revealed a significant prepulse intensity effect $(\mathrm{F}(4,112)=$ $19.55, p<0.0001)$. This supports the observation that the prepulse-induced reactivity increased as a function of increasing prepulse intensity. In addition, the analysis revealed a significant day effect $(\mathrm{F}(2,56)=7.08, p<0.0019)$ and a day $\times$ treatment interaction $(\mathrm{F}(4,56)=2.82, p<0.034)$.

As can be seen in Figure 4a and as was confirmed by post hoc comparisons, the mean reactivity elicited by the 

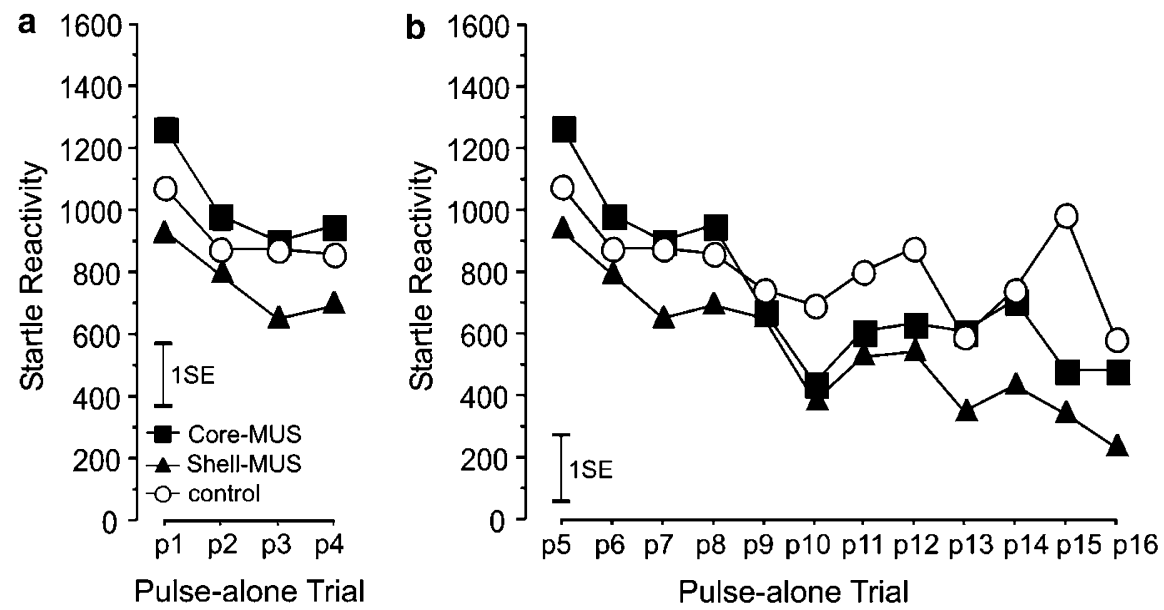

Figure 3 Effects of infusion of muscimol in either the core or the shell subregion on startle reactivity during the habituation phase (a) and the following 12 pulse-alone trials (b) on the infusion day only. The I SE bars indicate one standard error as derived from the ANOVA. Core-MUS, $n=9 ;$ Shell-MUS, $n=8$; control, $n=15$
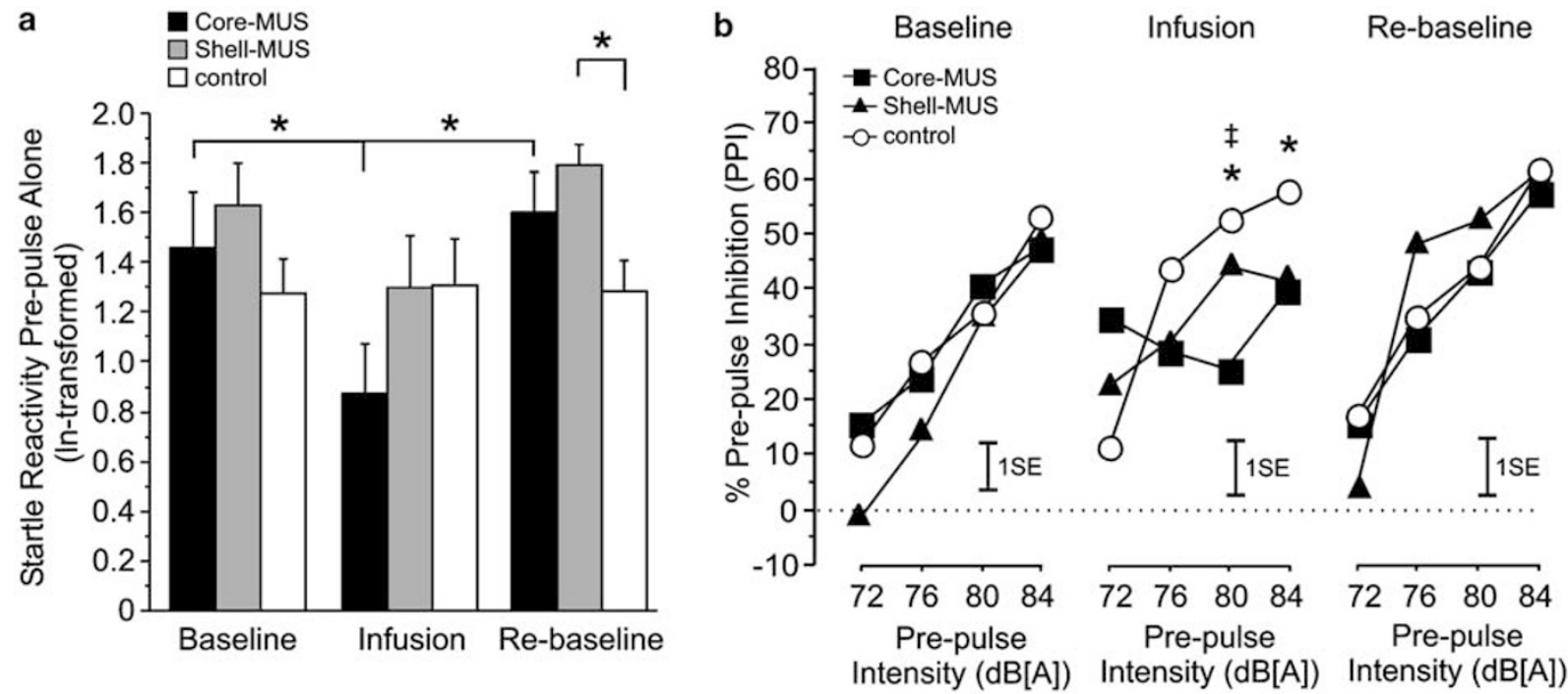

Figure 4 Effects of muscimol infusion in either the core or the shell subregion on the startle reactivity elicited by the prepulse alone presentations (a) and on the percentage prepulse inhibition (b). In (a), the asterisks indicates a significant difference $(p<0.05)$ as revealed by the post hoc analyses. In the middle panel of (b), the asterisks indicate significant differences in \%PPI at the specific prepulse intensity between the Core-MUS and the control group $(p<0.05)$. The symbol $\ddagger$ points to a significant difference in \%PPI between the Shell-MUS and the Core-MUS group. The I SE bars in (b) indicate one standard error as derived from the ANOVA. Core-MUS, $n=9$; Shell-MUS, $n=8$; control, $n=15$.

prepulse alone stimuli was significantly decreased in the Core-MUS on the infusion day in comparison to the baseline day $(p<0.013)$. On the re-baseline day, the startle reactivity shown by the Core-MUS group was fully restored in comparison to the infusion day $(p<0.004)$ and has reached a level comparable to that on the baseline day $(p>0.50)$. Infusion of muscimol into the shell did not decrease the startle reactivity in comparison to the baseline day $(p>0.17)$ (Figure 4a). A nearly significant increase in reactivity, however, could be observed in the Shell-MUS group on the re-baseline day in comparison to the infusion day $(p<0.053)$. The reactivity to the prepulse stimuli shown by the control group attained an equal level on all days.

On the baseline day as well as the infusion day itself, no significant treatment differences could be observed (baseline: $F(2,28)=0.91, p>0.41$, and infusion day: $\mathrm{F}(2,28)=1.26, p>0.29)$. The analysis of the re-baseline day, however, yielded a significant treatment effect $(\mathrm{F}(2,28)=3.39, p<0.049)$. Post hoc comparisons revealed that the reactivity shown by the Shell-MUS group was significantly increased in comparison to the control group $(p<0.021)$, while the Core-MUS did not differ from the control group $(p>0.05)$.

Prepulse inhibition (\%PPI). Analysis of the mean \%PPI across the three experimental days yielded a significant effect of prepulse intensity $(\mathrm{F}(3,84)=53.45, p<0.0001)$, indicating a gradual increase in the amount of inhibition as a function of the intensity of the prepulse stimulus (Koch, 1999). In addition, the analysis revealed a significant days $\times$ prepulse intensity interaction $(\mathrm{F}(6,168)=3.65$, $p<0.003)$ and a significant days $\times$ treatment $\times$ prepulse intensity interaction $(\mathrm{F}(12,168)=2.54, p<0.005)$. 
As depicted in Figure $4 \mathrm{~b}$, an increase in prepulse intensity resulted in a comparable increase in \%PPI on the baseline as well as on the re-baseline day. In addition, on each of the two days this increase was irrespective of treatment. These impressions were supported by separate analyses on each of the 3 days. On the baseline and re-baseline day, no significant treatment $\times$ prepulse type interaction (both $p>0.3$ ) was found. In contrast, infusion of muscimol affected this 'expected' increase in \%PPI as a function of increasing prepulse intensities, since on the infusion day the treatment $\times$ prepulse intensity interaction reached statistical significance $(\mathrm{F}(6,84)=5.00, p<0.0003)$.

Infusion of muscimol into the core resulted in complete loss of the prepulse intensity dependency of PPI. As shown in Figure $4 \mathrm{~b}$, the potency of the increasing prepulse intensities to attenuate the startle reactivity was diminished in the Core-MUS, while it was intact in the control group. These impressions were confirmed by supplementary post hoc comparisons for each of the prepulse intensities separately, which revealed a close to significance increase in \%PPI for the $72-\mathrm{dB}[\mathrm{A}]$ prepulse intensity in the CoreMUS in comparison to the control group $(p<0.073)$. Furthermore, an attenuation of \%PPI was observed in the Core-MUS in comparison to the control group, which approached significance for the 76- $\mathrm{dB}$ [A] prepulse intensity $(p<0.089)$. At the $80-$ as well as the $84-\mathrm{dB}[\mathrm{A}]$ prepulse intensity, a significant reduction of \%PPI in the Core-MUS group compared to the control group (both $p<0.04)$ was observed. At the $80-\mathrm{dB}[\mathrm{A}]$ prepulse intensity, the Core-MUS also showed an attenuation of \%PPI in comparison to the Shell-MUS group $(p<0.05)$. The ShellMUS group did not differ significantly from the controls at either of the prepulse-intensities.

\section{Effects of Muscimol Infusion on Locomotor Activity}

The analysis of the activity across the four experimental days revealed a significant 10 -min blocks effect $(F(8,232)=$ $174.25, p<0.0001$ ), which reflects a general reduction of activity, that is habituation, over the $90 \mathrm{~min}$ of testing. Its interaction terms with treatment $(\mathrm{F}(16,232)=2.04, p<$ $0.013)$ as well as with days and treatment $(F(48,696)=$ $1.51, p<0.017)$ attained significance. The analysis also yielded a significant day effect $(\mathrm{F}(3,87)=44.16, p<0.0001)$, and a significant treatment $\times$ day interaction $(F(6,87)=$ 14.90, $p<0.0001$ ).

On the baseline day, the treatment by 10-min blocks interaction was found to be significant $(\mathrm{F}(16,232)=2.28$, $p<0.005)$. Post hoc analysis revealed that this interaction was solely attributable to a reduction in activity during the eighth block of the Shell-MUS rats in comparison to the other groups (Shell-MUS $v s$ control: $p<0.04$, Shell-MUS $v s$ Core-MUS: $p<0.02$ ), which do not differ from each other (Core-MUS $v s$ control: $p>0.57$ ). During all the other 10-min blocks, the treatment groups did not differ from each other (all $p>0.05$, Figure 5).

On the infusion day, a substantial reduction in activity was observed in the Core-MUS group in comparison to the control and to the Shell-MUS groups. Infusion of muscimol in the shell did not alter the activity of these rats in

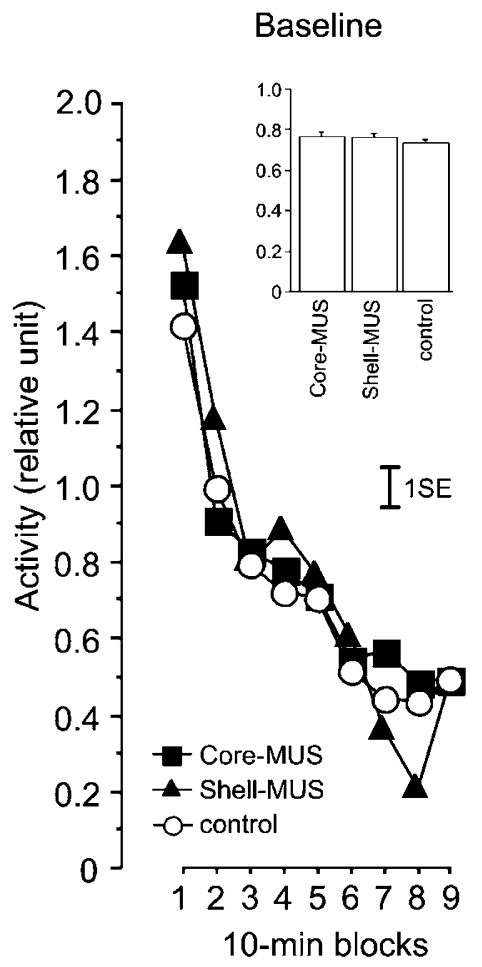

Infusion
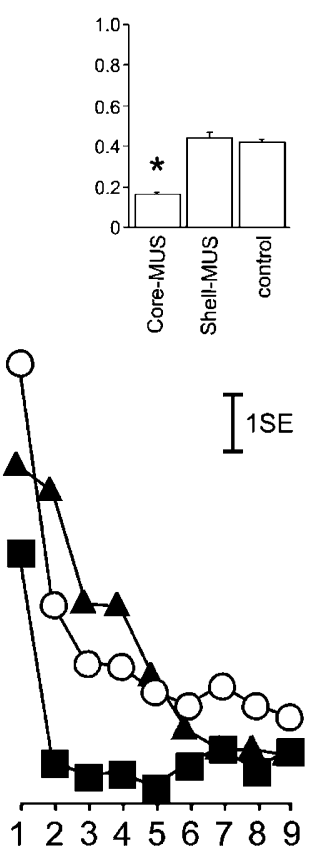

10-min blocks
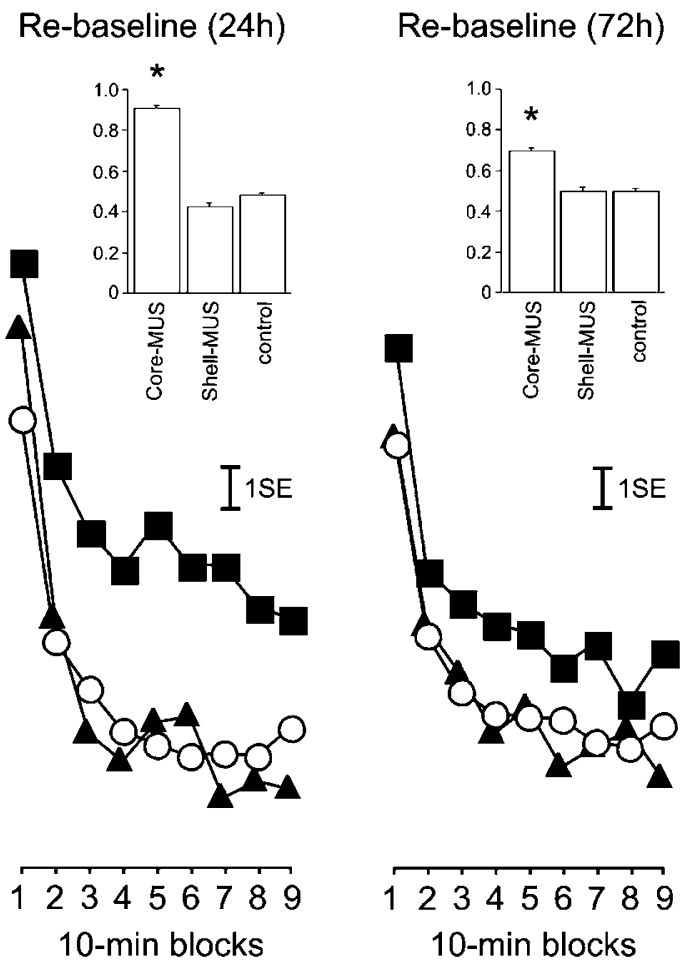

Figure 5 Effects of infusion of muscimol in either the core or the shell subregion on activity. The line plots depict the level of activity across the 90 min on different experimental days. The histogram inserts illustrate the mean level of activity per treatment group on different days. The asterisks indicate a significant difference $(p<0.05)$ of the Core-MUS group compared to the other groups as revealed by the post hoc analyses. The I SE bar indicates one standard error as derived from the ANOVA. Core-MUS, $n=11$; Shell-MUS, $n=7$; control, $n=14$. 
comparison to the control rats (Figure 5). This was supported by a significant main effect of treatment $(\mathrm{F}(2,29)=6.45, p<0.0048)$ and a significant treatment $\times$ 10 -min blocks interaction $(\mathrm{F}(16,232)=1.94, p<0.019)$. Post hoc analysis confirmed these observations by revealing that the treatment effect was solely attributable to the decrease in activity in the Core-MUS group compared to the control group and the Shell-MUS group (Core-MUS vs control: $p<0.003$, Core-MUS vs Shell-MUS: $p<0.012$ ), which did not differ from each other (Shell-MUS $v s$ control: $p>0.88$ ).

On re-baseline day 1 ( $24 \mathrm{~h}$ after the infusion of muscimol), the Core-MUS group showed an overall increase of activity compared with the Shell-MUS and the control groups (Figure 5). This increase in total activity was almost two-fold in comparison to the other groups (Core-MUS: $0.91 \pm 0.02$, Shell-MUS: $0.46 \pm 0.02$, and control: $0.46 \pm 0.01$, relative units, mean $\pm S E M)$. These impressions were supported by a main effect of treatment $(\mathrm{F}(2,29)=17.86$, $p<0.0001)$. Post hoc analysis revealed that the treatment effect can only be ascribed to the increase in activity in the Core-MUS group compared to the control group and the Shell-MUS group (Core-MUS vs control: $p<0.0001$, CoreMUS vs Shell-MUS: $p<0.0001)$, which did not differ from each other (Shell-MUS $v s$ control: $p>0.917$ ).

On re-baseline day 2 ( $72 \mathrm{~h}$ after infusion), the same observations were made as on re-baseline day 1 . Although the effect was less pronounced, again the Core-MUS group showed an enhanced activity in comparison to the ShellMUS and control groups (see Figure 5). This was confirmed by the $3 \times 9$ ANOVA, which yielded a main effect of treatment $(\mathrm{F}(2,29)=5.03, p<0.014)$. Again pairwise post hoc comparisons confirmed that the treatment effect was solely attributable to the hyperactivity shown by the CoreMUS group (Core-MUS vs control: $p<0.009$, Core-MUS vs Shell-MUS: $p<0.015)$, which did not differ from each other (Shell-MUS vs control: $p>0.79$ ).

\section{Spread of Muscimol}

The distribution of the infused $\left[{ }^{3} \mathrm{H}\right]$ muscimol into the core and the shell was typically radial in shape (see Figure $6 \mathrm{c}$ and $\mathrm{d}$, respectively). Superimposition of the labeled area obtained from the autoradiogram onto the cresyl violet counterstained section revealed that the spread of the labeled muscimol into the neighboring accumbal subregion was minimal (Figure $6 c$ and $\mathrm{f}$ ). These observations were supported by quantitative measures of the optical density, the width, the height, and the surface area of the labeled signal. The radioactivity profiles obtained after measurement of the optical density revealed that the radioactivity levels, after infusion into the core as well as the shell, declined progressively as the distance from the injection site increased along the rostrocaudal axis. As can be seen in Figure $6 \mathrm{~g}$, the mean rostrocaudal extent of diffusion after muscimol infusion into the core was comparable to that observed after infusion into the shell (around $1.0 \mathrm{~mm}$ from the injection site). Given that beyond $1.1 \mathrm{~mm}$ from the injection site the signal fell within the noise level of the detection method (which made proper delineation of the labeled area not feasible), no reliable autoradiographic measures could be evaluated beyond this rostrocaudal extent.
As shown in Figure $6 \mathrm{~h}$, the width of the labeled area progressively decreased in both groups, as a function of the distance from the injection site along the rostrocaudal axis. Similar inversed relationships were observed with respect to the height and the surface area of the labeled region (data not shown). Both measures decreased as the distance from the injection site along the rostrocaudal axis increased. The mean height of the labeled area after infusion into the core varied from $3.7 \mathrm{~mm}$ at the injection site to $2.3 \mathrm{~mm}$ at $1.1 \mathrm{~mm}$ from the injection site. The mean height after infusion in the shell ranged from $3.5 \mathrm{~mm}$ (at the injection site) to $1.8 \mathrm{~mm}$ (at $1.1 \mathrm{~mm}$ from the injection site). The mean surface area ranged from $4.3 \mathrm{~mm}^{2}$ (at the injection site) to $2.2 \mathrm{~mm}^{2}$ (at $1.1 \mathrm{~mm}$ from the injection site) when the radiolabeled muscimol was infused in the core, and $4.6 \mathrm{~mm}^{2}$ (at the injection site) to $2.2 \mathrm{~mm}^{2}$ (at $1.1 \mathrm{~mm}$ from the injection site) when it was infused into the shell.

\section{DISCUSSION}

The present study demonstrates that temporary inactivation of the core or the shell subregions of the nucleus accumbens with muscimol was not associated with equivalent effects on PPI and activity. The key findings are: first, inactivation of the core but not of the shell resulted in a complete loss of the prepulse intensity dependency of PPI when compared to the control group. These effects on PPI were associated with a significant reduction in response to the prepulse stimuli in the core group, without a concomitant effect on startle reactivity elicited by the pulse-alone stimuli. Second, inactivation of the core in contrast to the shell led to a significant reduction in activity in comparison to the control group. In addition, 24 and $72 \mathrm{~h}$ after muscimol infusion, the core infused rats were hyperactive in comparison to the control and the shell infused groups. Hence, our data lend support to the view of a functional differentiation between the core and the shell subregions of the nucleus accumbens by showing that the core is preferentially involved in PPI and locomotion.

\section{Methodological Considerations}

One prerequisite for a successful functional dissociation between the two accumbens subregions using intracerebral microinfusions is to prevent the infused drug from spreading into the other subregion. Several measures have been taken to preclude this in the present study. First, the location of the guide cannulae to inactivate the shell subregion was chosen in such a manner (in the 'medial' part of the shell) that mechanical damage to the core subregion itself was prevented. Furthermore, infusion into the 'medial' shell circumvented possible diffusion of the drug dorsally into the core, as most likely would have occurred if the muscimol had been applied to the 'ventral' shell. As a consequence of these methodological considerations, we were not able to inactivate the entire shell area. Yet, one could argue whether this is practically feasible at all since the shell is an arch shaped structure, and as a consequence it would require multiple infusions for complete inactivation. Second, the volume of the infused solution was considerably smaller $(0.2 \mu \mathrm{l})$ than commonly 

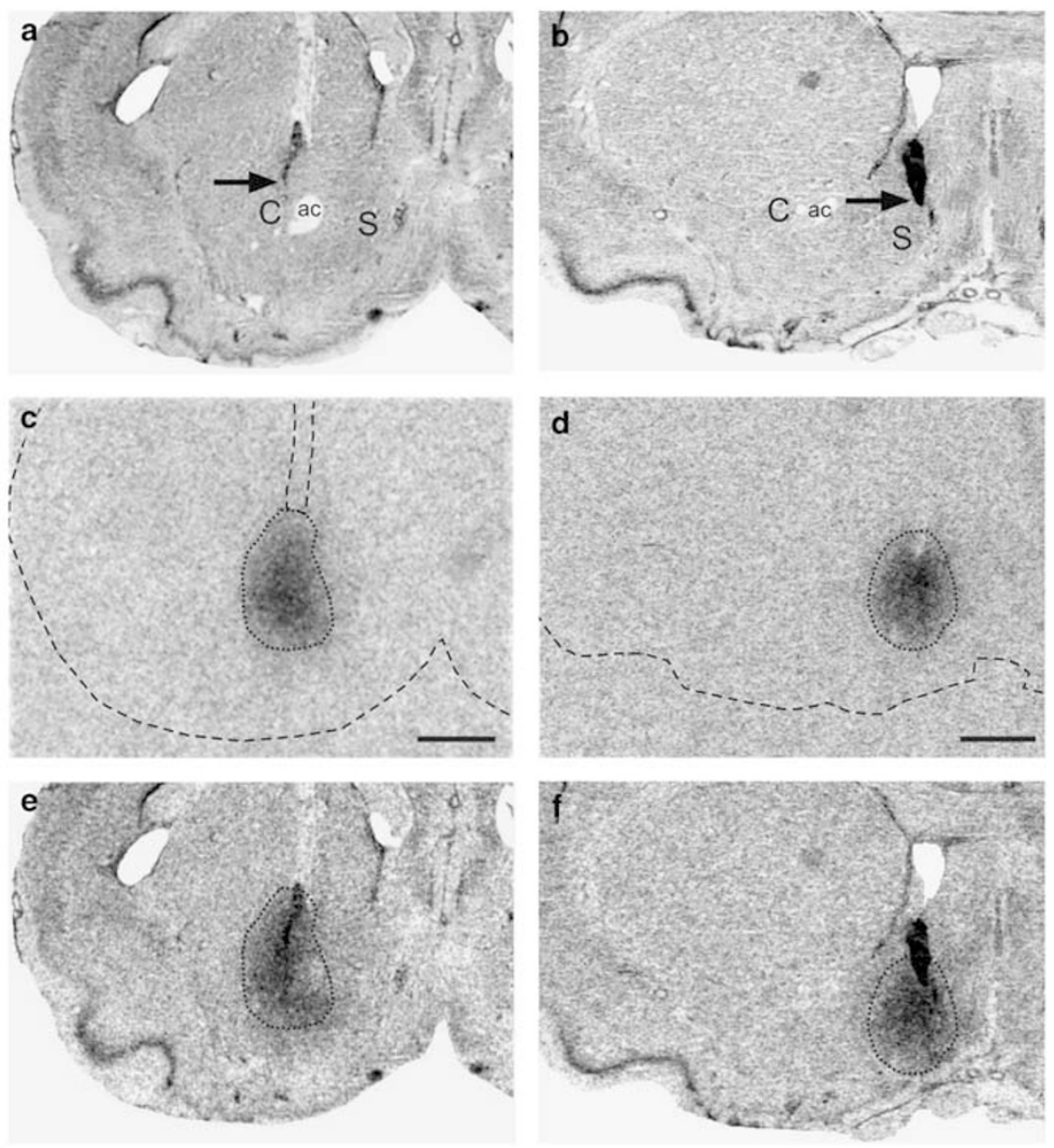

g

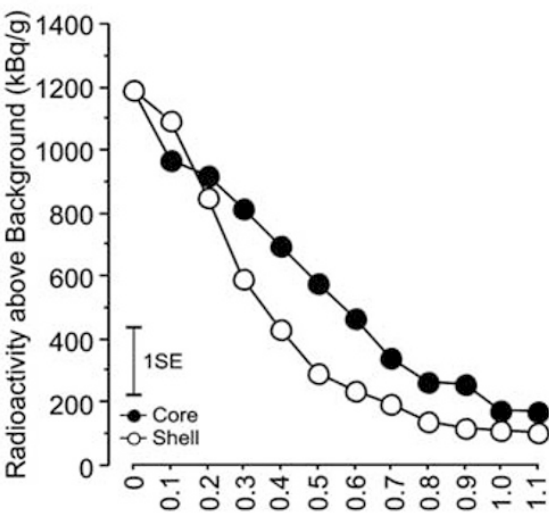

Distance from Injection Site $(\mathrm{mm})$ $\mathrm{h}$

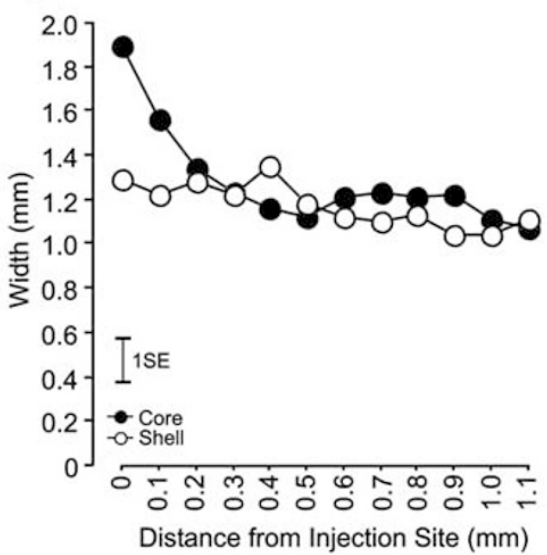

Figure 6 Spread of muscimol in the core and shell subregions of the nucleus accumbens. Panels (a) and (b) depict a photomicrograph of a cresyl violet stained section of a core- and shell-implanted brain, respectively. In (c) and (d), a photograph of the autoradiogram obtained after infusion of the $\left.{ }^{3} \mathrm{H}\right]$ labeled muscimol into the section depicted in panels (a) and (b), respectively, is shown. The dotted lines indicate the designated border of radiolabeled area used to determine the optical density. The dashed lines point to the relative position of the sections depicted in panels (a) and (b). Panels (e) and ( $f$ ) represent a photomicrograph of the autoradiogram depicted in (c) and (d) when superimposed on the cresyl violet stained section of (a) and (b), illustrating the relative spread (indicated with the dotted line) of the radiolabeled muscimol in the core and the shell, respectively. The line plot in (g) shows the radioactivity profile along the rostrocaudal axis $15 \mathrm{~min}$ after infusion of the radiolabeled muscimol $(0.2 \mu \mathrm{l})$ infused into either the core or the shell. Values are shown after subtraction of the background optical density. The line plot in $(\mathrm{h})$ depicts the decline of the mean width of the labeled area as a function of the rostrocaudal distance from the injection site after infusion of radiolabeled muscimol into either the core or the shell. The I SE bars in (g) and (h) indicate one standard error. The scale bar in (c) applies to (a) and (e), while the scale bar in (d) also applies to (b) and (f). They both represent I.0 mm. Abbreviations: ac, anterior commissure; $C$, core subregion; $\mathrm{S}$, shell subregion. The arrows in (a) and (b) point to the location of the injection site. 
used in drug-infusion studies $(0.5 \mu \mathrm{l}$ or larger) to selectively stimulate or inactivate either one of the accumbal subregions (eg Maldonado-Irizarry and Kelley, 1994; Reijmers et al, 1995) or to investigate functional segregations within one accumbal subregion (eg Reynolds and Berridge, 2003; Zhang and Kelley, 2000). Although sparse data are available on quantification of muscimol diffusion after intracerebral microinfusions in the rodent brain, they do suggest that the diffusion of muscimol is larger than initially assumed (Edeline et al, 2002; Martin, 1991).

Our radioactive muscimol infusion experiment demonstrates that an injected volume of $0.2 \mu \mathrm{l}$ is sufficient to lead to a diffusion of approximately $1.0 \mathrm{~mm}$ from the injection site in one direction of the rostrocaudal axis, when infused into the core or the shell. In addition, it gives rise to a labeled area with a mean width of 1.9 or $1.3 \mathrm{~mm}$ at the injection site when infused into either the core or the shell, respectively (notably, the mean width of the core and the shell as determined from Figures 11 and 12 of Paxinos and Watson's Rat Brain Atlas (1997) is approximately 1.8 and $1.0 \mathrm{~mm}$, respectively). These findings are in accordance with Martin (1991) and Edeline et al (2002), who obtained comparable values of muscimol diffusion after infusion into the cortex, the nucleus basalis magnocellularis and the thalamic reticular nucleus. Hence, our findings indicate that although the use of an injected volume of $0.2 \mu \mathrm{l}$ already produced a considerable diffusion radius, it nonetheless did not exceed the anatomical borders of either the core or the shell.

Although it was not our aim to investigate the effects of increasing infused volumes on the extent of diffusion in the nucleus accumbens (the initial objective was to quantify the diffusion radius employing the same injected volume as for the behavioral studies), our data do suggest that the use of any injected volume that is considerably larger (eg $0.5 \mu \mathrm{l}$ ) than the one used in the present study, almost certainly will result in spread of muscimol into the other accumbal subregion. Our findings, therefore, raise doubts about the specificity of the behavioral effects observed in the studies that have employed such large injected volumes (eg Maldonado-Irizarry and Kelley, 1994; Reijmers et al, 1995; Reynolds and Berridge, 2003; Zhang and Kelley, 2000).

\section{Effects of Muscimol Infusion in the Core or the Shell on PPI and Startle}

No significant treatment effect and no significant interactions of treatment with the other variables on the startle reactivity were found during the four habituation pulses, or the following 12 pulse-alone trials. Thus, it can be ruled out that the irregularities in PPI seen following muscimol infusion into the core are due to changes in startle amplitude. The lack of an effect of shell inactivation on startle shows, in contrast with previous findings, that excitotoxic lesions of the shell resulted in an increased startle reactivity in comparison to core-lesions and the control group (Jongen-Rêlo et al, 2002; Kodsi and Swerdlow, 1997).

Inactivation of the core resulted in a complete loss of prepulse dependency of PPI in comparison with the control group, as the \%PPI did not increase with increasing prepulse intensities. This effect was accompanied by a reduction in reactivity on the prepulse alone trials, which was not observed in the control group. Unlike the effects of core/shell inactivation or lesions on PPI, the effects of these manipulations on reactivity to the prepulse stimulus have not been reported before. However, we do want to emphasize that this reduced reactivity on prepulse alone trials can have important implications and deserves special attention in further studies (see also Yee et al, 2004a, b). As it has previously been shown in mice, disruptions of PPI by pharmacologically distinct classes of drugs can be differentiated according to the effects on prepulse elicited reactivity (Yee et al, 2004a,b). Whereas a PPI disrupting systemic injection of the dopamine agonist apomorphine enhanced the reactivity to the prepulse stimuli (Yee et al, 2004b), a PPI disrupting systemic injection of the NMDA receptor antagonists dizocilpine (MK-801) or phencyclidine (PCP) tended to reduce this reactivity (Yee et al, 2004a). Assuming that the neuropsychopharmacology of PPI is similar in rats and mice, the observed reduction in reactivity on the prepulse alone trials together with the loss of prepulse dependency of PPI after inactivation of the core, is strikingly similar to the effects observed after manipulations of the glutamatergic system as described by Yee et al (2004a). This interesting observation might be indicative of a common neural substrate underlying the disruption of PPI seen after inactivation of the core subregion, and NMDA receptor antagonism following systemic manipulations, and therefore encourages further investigation into the relationship between PPI, prepulse elicited reactivity, and the core subregion.

The findings of the present study show that inactivation of the shell subregion, in contrast to inactivation of the core, does not result in changes in \%PPI as a function of the prepulse intensity compared with the control group. It is important to note that we have made similar observations in a previous study employing selective excitotoxic lesions of the core or the shell, which findings favored a core-lesion over a shell-lesion induced PPI deficit (Jongen-Rêlo et al, 2002). Hence, our findings all together are not in complete accordance with suggestions of Swerdlow and co-workers, who proposed that both subregions are involved in the regulation of PPI (Kodsi and Swerdlow, 1997; Wan et al, 1994; Wan and Swerdlow, 1996).

It should be noted, however, that the present study is the first to investigate the effects of pharmacological stimulation of the GABAergic system in the nucleus accumbens on PPI. Even though most studies in the past have demonstrated the importance of the accumbens dopaminergic system (for a review see Swerdlow et al, 2001), our present findings clearly suggest a function of the accumbal GABAergic system in the regulation of PPI. Moreover, this regulatory function can almost certainly be attributed to the core rather than to the shell subregion. However, we cannot exclude the possibility that the shell might exert a regulatory role on PPI via other neurotransmitter systems such as dopamine and glutamate, as proposed by Wan et al (1994) and Wan and Swerdlow (1996). Yet, we believe that the remarkable resemblance between our present (using inactivation via muscimol) and our previous findings (employing selective permanent lesions) does indicate that the role of the shell in the regulation of PPI, if existent, is minor compared to that of the core. This later observation 
is in contrast to the regulation of latent inhibition in which both subregions appear to be involved (Jeanblanc et al, 2002; Weiner et al, 1996, for a review see Weiner and Feldon, 1997).

\section{Effects of Muscimol Infusion in the Core or the Shell on Activity}

Our findings clearly demonstrate a functional dissociation in the nucleus accumbens with regard to the modulation of locomotion. While inactivation of the core resulted in clear changes in the level of activity of the rats, inactivation of the shell was found to be ineffective. This functional difference between the core and the shell in the modulation of locomotion can be explained by the distinct anatomical connections of the two subregions. In contrast to the shell subregion, the core sends efferent projections to 'typical' basal ganglia motor output structures such as the dorsolateral ventral pallidum, subthalamic nucleus, endopenducular nucleus, and substantia nigra, which in turn project via the thalamus to the premotor areas and eventually the primary motor areas (Heimer et al, 1991; Zahm and Brog, 1992). Several studies in rodents have pointed out that the projection of the nucleus accumbens to the ventral pallidum is GABAergic in nature (Mogenson et al, 1980; Mogenson et al, 1983). Pharmacological manipulations of this inhibitory pathway have demonstrated that this projection is essential for the modulation of locomotor behavior (eg Jones et al, 1981; Mogenson and Nielsen, 1983; Swerdlow and Koob, 1987). Hence, we hypothesize that stimulation of the $\mathrm{GABA}_{\mathrm{A}}$ receptors by infusing muscimol into the core subregion will lead to a reduced firing of the neurons present in the core. One of the pathways that might be affected by this core inactivation is the GABAergic output to the ventral pallidum, which exhibits reduced activity as a consequence of inactivation of the core. This would lead to disinhibition of neurons in the ventral pallidum. Consequently, the GABAergic projection of the ventral pallidum to more downstream motor circuitry areas is less inhibited, eventually leading to a decrease in locomotion mediated by this pathway.

The present findings confirm previous reports using either excitotoxic lesions of the core or the shell or NMDA-antagonist microinfusions into the two subregions (Maldonado-Irizarry and Kelley, 1994, 1995; Parkinson et al, 1999). However, they are not in accordance with previous lesion findings, which suggest a preferential involvement of the shell subregion in locomotor activity (Jongen-Rêlo et al, 2002; Weiner et al, 1996). A satisfying explanation for this latter discrepancy is difficult to give, but might be found in the method of inactivation (pharmacological vs lesions), activity assessment (open field $v s$ activity chambers), or recovery time allowed (see also Jongen-Rêlo et al, 2002, pp 104). Our findings indeed appear to suggest that the time point of behavioral assessment can be crucial in order to detect an effect on activity after manipulations of the nucleus accumbens. In the present study, we observed a massive decrease in activity of the rats immediately after muscimol infusion into the core to the extent that the rats were akinetic. At 1 day and even 3 days later, the core infused rats were significantly hyperactive in comparison to the control and the shell infused rats, without having received any manipulation during this period.

Interestingly, similar observations have been made by Maldonado-Irizarry and co-workers (1995) after excitotoxic lesions of the core subregion. In their study, the corelesioned rats were found to be (according to an informal observation) akinetic as well, the first few days after surgery (Maldonado-Irizarry and Kelley, 1995, pp 558). The activity level of the shell-lesioned rats on the other hand appeared to be comparable to that of the controls. In contrast, 1 and 3 weeks after surgery, when the rats were tested in activity cages, the core-lesioned rats were hyperactive when compared to the control and the shell-lesioned rats. Although Maldonado-Irizarry and co-workers have made their observations after excitotoxic lesions of the core subregion and not temporary inactivation, the similarities between the results of both studies are striking.

At present, we can only speculate on why the hyperactivity of the core infused animals emerges one day after the infusion and persists for at least another two additional days. One could argue that the long-lasting hyperactivity is the result of permanent damage of the core region caused by the muscimol infusion. We have reason to assume that this is not likely to be the case, since all rats were tested in a novel environment (ie a shuttle box apparatus wherein the number of shuttle box crossings can be regarded as a measure of activity) 6 days after the last activity measurement and no differences in activity were observed between the original infusion groups (unpublished observation, Pothuizen and Feldon, 2004). Hence, the hyperactivity seen in the core-infused rats was most likely not permanent.

We hypothesized above that muscimol-induced inhibition of the GABAergic output of the core to the ventral pallidum is responsible for the substantial reduction in locomotion observed immediately after the infusion. If this hypothesis is correct, then we would propose that the hyperactivity observed 24 and $72 \mathrm{~h}$ after the infusion might be caused by compensatory mechanisms taking place either in the nucleus accumbens or the ventral pallidum. Although highly speculative, one example of these compensatory mechanisms could be the upregulation or enhanced sensitivity of GABA receptors located postsynaptically on ventral pallidum neurons due to the denervation of the GABAergic input from the core. As a consequence, these ventral pallidum neurons are more inhibited after restoration of the GABAergic input of the core. This augmented inhibition of the ventral pallidum neurons might result in a reduced GABAergic outflow to the motor output structures downstream of the circuitry, resulting in the development of enhanced locomotor activity. At the same time, other neurotransmitter systems within the nucleus accumbens, such as the dopaminergic system, can exert concomitant effects on the altered accumbal GABAergic system. Notably, damage to this neurotransmitter system as well as stimulation via dopaminergic agonists infused into the accumbens has been shown to result in enhanced locomotor activity (eg Boye et al, 2001; Jones et al, 1981; Parkinson et al, 1999; Sellings and Clarke, 2003; but see Taghzouti et al, 1985).

In conclusion, our findings are, to our knowledge, the first to show a functional dissociation between the core and the shell after GABAergic pharmacological manipulations with microinfusions of muscimol into these two brain 
regions when testing on PPI and locomotor activity. Our data indicate that, in contrast to the shell subregion, the core plays a dominant role in sensorimotor gating. Inactivation of the core did not only affect PPI, but was also associated with a reduction in prepulse stimulus reactivity. In addition, our data reveal that the core in contrast to the shell plays an important role in the regulation of locomotion. It may exert this regulatory role via its inhibitory GABAergic efferent projection to the basal ganglia motor output structures.

\section{ACKNOWLEDGEMENTS}

We would like to thank the animal facility team for the excellent care of the animals, Mr Christiaan Schlatter for preparation of the guide cannulae, Mr Peter Schmid for his technical assistance, Ms Liz Weber for preparing the histology, Dr Benjamin K Yee for critically reading the manuscript, and Mrs Misa Kuper-Yamanaka for her editorial assistance. This study was funded by the Swiss Federal Institute of Technology, Zurich. The research was supported by the Swiss Federal Institute of Technology Zurich, Grant Number TH-13./00-2.

\section{REFERENCES}

Bast T, Feldon J (2003). Hippocampal modulation of sensorimotor processes. Prog Neurobiol 70: 319-345.

Bast T, Zhang W-N, Feldon J (2001). The ventral hippocampus and fear conditioning in rats. Differential anterograde amnesias of fear after tetrodotoxin inactivation and infusion of the GABAa agonist muscimol. Exp Brain Res 139: 39-52.

Boye SM, Grant RJ, Clarke PB (2001). Disruption of dopaminergic neurotransmission in nucleus accumbens core inhibits the locomotor stimulant effects of nicotine and D-amphetamine in rats. Neuropharmacology 40: 792-805.

Brog JS, Salyapongse A, Deutch AY, Zahm DS (1993). The pattern of afferent innervation of the core and the shell in the 'accumbens' part of the ventral striatum: immunohistochemical detection of retrogradely transported fluoro-gold. J Comp Neurol 338: 255-278.

Deutch AY, Cameron DS (1992). Pharmacological characterization of dopamine systems in the nucleus accumbens core and shell. Neuroscience 46: 49-56.

Edeline J-M, Hars B, Hennevin E, Cotillon N (2002). Muscimol diffusion after intracerebral microinjections: a reevaluation based on electrophysiological and autoradiographic quantification. Neurobiol Learn Mem 78: 100-124.

Groenewegen HJ, Wright CI, Beijer AVJ, Voorn P (1999). Convergence and segregation of ventral striatal inputs and outputs. Ann NY Acad Sci 877: 49-63.

Heimer L, Zahm DS, Churchill L, Kalivas PW, Wohltmann C (1991). Specificity in the projection patterns of accumbal core and shell in the rat. Neuroscience 41: 89-125.

Hoffman HS, Searle JR (1965). Acoustic variables in the modification of startle reaction in the rat. J Comp Exp Psychol 60: 53-58.

Jeanblanc J, Hoeltzel A, Louilot A (2002). Dissociation in the involvement of dopaminergic neurons innervating the core and shell subregions of the nucleus accumbens in latent inhibition and affective perception. Neuroscience 111: 315-323.

Johnson K, Churchill L, Klitenick MA, Stacy Hook M, Kalivas PW (1996). Involvement of the ventral tegmental area in locomotion elicited from the nucleus accumbens or ventral pallidum. J Pharmacol Exp Ther 277: 1122-1131.
Jones DL, Mogenson GJ, Wu M (1981). Injections of dopaminergic, cholinergic, serotonergic and GABAergic drugs into the nucleus accumbens: effects on locomotor activity in the rat. Neuropharmacology 20: 29-37.

Jones SR, Odell SJ, Marshall JF, Wightman RM (1996). Functional and anatomical evidence for different dopamine dynamics in the core and shell of the nucleus accumbens in slices of rat brain. Synapse 23: 224-231.

Jongen-Rêlo AL, Groenewegen HJ, Voorn P (1993). Evidence for a multicompartmental histochemical organization of the nucleus accumbens in the rat. J Comp Neurol 337: 267-276.

Jongen-Rêlo AL, Kaufmann S, Feldon J (2002). A differential involvement of the shell and core subterritories of the nucleus accumbens of rats in attentional processes. Neuroscience 111: 95-109.

Jongen-Rêlo AL, Kaufmann S, Feldon J (2003). A differential involvement of the shell and core subterritories of the nucleus accumbens of rats in memory processes. Behav Neurosci 117: $150-168$.

Jongen-Rêlo AL, Voorn P, Groenewegen HJ (1994). Immunohistochemical characterization of the shell and core territories of the nucleus accumbens in the rat. Eur J Neurosci 6: 1255-1264.

Koch M (1999). The neurobiology of startle. Prog Neurobiol 59: 107-128.

Kodsi MH, Swerdlow NR (1994). Quinolinic acid lesions of the ventral striatum reduce sensorimotor gating of acoustic startle in rats. Brain Res 643: 59-65.

Kodsi MH, Swerdlow NR (1997). Reduced prepulse inhibition after electrolytic lesions of nucleus accumbens subregions in the rat. Brain Res 773: 45-52.

Kretschmer BD, Koch M (1998). The ventral pallidum mediates disruption of prepulse inhibition of the acoustic startle response induced by dopamine agonists, but not by NMDA antagonists. Brain Res 798: 204-210.

Maldonado-Irizarry CS, Kelley AE (1994). Differential behavioral effects following microinjection of an NMDA antagonist into nucleus accumbens subregions. Psychopharmacology 116: 65-72.

Maldonado-Irizarry CS, Kelley AE (1995). Excitoxic lesions of the core and shell subregions of the nucleus accumbens differentially disrupt body weight regulation and motor activity in rat. Brain Res Bull 38: 551-559.

Martin JH (1991). Autoradiographic estimation of the extent of reversible inactivation produced by microinjection of lidocaine and muscimol in the rat. Neurosci Lett 127: 160-164.

Martin JH, Ghez C (1999). Pharmacological inactivation in the analysis of the central control of movement. J Neurosci Methods 86: $145-159$.

Mogenson GJ, Jones DL, Yim CY (1980). From motivation to action: functional interface between the limbic system and the motor system. Progr Neurobiol 14: 69-97.

Mogenson GJ, Nielsen MA (1983). Evidence that an accumbens to subpallidal GABAergic projection contributes to locomotor activity. Brain Res Bull 11: 309-314.

Mogenson GJ, Swanson LW, Wu M (1983). Neural projections from nucleus accumbens to globus pallidus, substantia innominata, and lateral preoptic-lateral hypothalamic area: an anatomical and electrophysiological investigation in the rat. J Neurosci 3: 189-202.

O’Donnell P, Grace AA (1993). Physiological and morphological properties of accumbens core and shell neurons recorded in vitro. Synapse 13: 135-160.

Parkinson JA, Olmstead MC, Burns LH, Robbins TW, Everitt BJ (1999). Dissociation in effects of lesions of the nucleus accumbens core and shell on appetitive pavlovian approach behavior and the potentiation of conditioned reinforcement and locomotor activity by D-amphetamine. J Neurosci 19: 2401-2411. 
Paxinos G, Watson C (1997). The Rat Brain in Stereotaxic Coordinates Compact, 3rd edn. Academic Press: San Diego, CA.

Pulvirenti L, Berrier R, Kreifeldt M, Koob GF (1994). Modulation of locomotor activity by NMDA receptors in the nucleus accumbens core and shell regions of the rat. Brain Res 664: 231-236.

Reijmers LGJE, van der Heyden PML, Peeters BWMM (1995). Changes in prepulse inhibition after local administration of NMDA receptor ligands in the core region of the rat nucleus accumbens. Eur J Pharmacol 272: 131-138.

Reynolds SM, Berridge KC (2003). Glutamate motivational ensembles in nucleus accumbens: rostrocaudal shell gradients of fear and feeding. Eur J Neurosci 17: 2187-2200.

Sellings LH, Clarke PB (2003). Segregation of amphetamine reward and locomotor stimulation between nucleus accumbens medial shell and core. J Neurosci 23: 6295-6303.

Stratford TR, Kelley AE (1997). GABA in the nucleus accumbens shell participates in the central regulation of feeding behavior. J Neurosci 17: 4434-4440.

Swerdlow NR, Geyer MA, Braff DL (2001). Neural circuit regulation of prepulse inhibition of startle in the current knowledge and future challenges. Psychopharmacology 156: 194-215.

Swerdlow NR, Koob GF (1987). Lesions of the dorsomedial nucleus of the thalamus, medial prefrontal cortex and pedunculopontine nucleus: effects on locomotor activity mediated by nucleus accumbens-ventral pallidal circuitry. Brain Res 412: 233-243.

Taghzouti K, Simon H, Louilot A, Herman JP, Le Moal M (1985). Behavioral study after local injection of 6-hydroxydopamine into the nucleus accumbens in the rat. Brain Res 344: 9-20.

Wan F-J, Geyer MA, Swerdlow NR (1994). Accumbens D2 modulation of sensorimotor gating in rats: assessing anatomical localization. Pharmacol Biochem Behav 49: 155-163.
Wan F-J, Geyer MA, Swerdlow NR (1995). Presynaptic dopamineglutamate interactions in the nucleus accumbens regulate sensorimotor gating. Psychopharmacology 120: 433-441.

Wan FJ, Swerdlow NR (1996). Sensorimotor gating in rats is regulated by different dopamine-glutamate interactions in the nucleus accumbens core and shell subregions. Brain Res 722: 168-176.

Weiner I, Feldon J (1997). The switching model of latent inhibition: an update of neural substrates. Behav Brain Res 88: $11-25$.

Weiner I, Gal G, Rawlins JNP, Feldon J (1996). Differential involvement of the shell and core subterritories of the nucleus accumbens in latent inhibition and amphetamine-induced activity. Behav Brain Res 81: 123-133.

Yee BK, Chang DT, Feldon J (2004a). The effects of dizocilpine and phencyclidine on prepulse inhibition of the acoustic startle reflex and on prepulse-elicited reactivity in C57BL6 mice. Neuropsychopharmacology 29: 1865-1877.

Yee BK, Russig H, Feldon J (2004b). Apomorphine-induced prepulse inhibition disruption is associated with a paradoxical enhancement of prepulse stimulus reactivity. Neuropsychopharmacology 29: 240-248.

Záborszky L, Alheid GF, Beinfeld MC, Eiden LE, Heimer L, Palkovitz M (1985). Cholecystokinin innervation of the ventral striatum: a morphological and radioimmunological study. Neuroscience 14: 427-453.

Zahm DS, Brog JS (1992). On the significance of subterritories in the 'accumbens' part of the ventral striatum. Neuroscience 50: 751-767.

Zahm DS, Heimer L (1990). Two transpallidal pathways originating in the rat nucleus accumbens. J Comp Neurol 302: 437-446.

Zhang M, Kelley AE (2000). Enhanced intake of high-fat food following striatal mu-opioid stimulation: microinjection mapping and Fos expression. Neuroscience 99: 267-277. 